\title{
Peer reviews of teaching as appreciative inquiry: Learning from 'the best' of our colleagues
}

\begin{abstract}
Research on peer review to date has focused on its role as a formal compliance mechanism, a process for enhancing and developing teaching practice, and as a considerable source of anxiety for educators. In this paper, we draw on scholarly reflections from our experience of undertaking a reciprocal, formative peer review in an Australian higher education setting. Our findings provide novel conceptual, empirical and practical insights by providing the first application of an appreciative inquiry framework to the process of peer review. We argue that adopting an appreciative inquiry framework assists us in learning from 'the best' aspects of our colleagues' teaching. Moreover, it offers a framework for understanding and responding to some of the challenges long-associated with peer review. In doing so, it presents potential benefits pertaining to student retention and learning outcomes, while opening up new possibilities for researching and practicing peer review.
\end{abstract}

\section{Keywords}

Peer review, learning and teaching, appreciative inquiry, higher education, scholarly reflection

\section{Introduction}

Peer review (PR) of teaching is now embedded in professional development and appraisal in higher education. Indeed, PR has become so central that it is mandated as part of promotion applications in some higher education settings (Crisp et al, 2009). To date, considerable literature has examined the function, purpose, benefits and limitations of PR (Bell \& Mladenovic, 2008; Gosling, 2014; Hamersley-Fletcher \& Orsmond, 2005; Peel, 2005). Much of this literature illustrates how PR may foster reflexivity within teaching practice. As the use of reflexivity has been identified as a component of 'best practice' pedagogy (Biggs \& Tang, 2011), it is essential that we critically reflect on our experiences to progress and develop our teaching and learning practices (Porter et al., 2020). In this article we therefore provide reflections on our own experiences conducting reciprocal PR in order to develop and advance research and practice in this area.

In doing so, one key finding from our experiences was that PR can constitute a form of appreciative inquiry (AI). As Watkins et al. (2011, p. 22) explain, “appreciative inquiry" focuses on the generative and life-giving forces in [a] system that are the things we want to increase". Drawing on these experiences, we suggest framing PR as a form of AI can be productive given the negative connotations and anxieties that often accompany the PR process (Purvis et al, 2009). As De Lautour (2009, p. 85) reflects, "it often takes someone else to articulate and recognize the strengths of one's practice". Rather than a process of appraisal, judgement and negative critique, in our own experience(s) PR largely functioned to highlight 'what we do well' and to draw out examples of best practice in our teaching.

In examining our experiences undertaking an AI-inflected process of PR, our article contributes to higher education research and practice in several ways. As the first research examining the applicability of AI principles to PR of teaching, this work fills a key lacuna in research examining the use of AI within higher education. Our findings demonstrate how an AI approach may overcome some of the challenges associated with PR identified in previous research. In doing so, they demonstrate AI's potential as a framework for understanding - and reconceptualizing - the role and function of PR in higher education. In turn, this work paves 
the way for a new area of empirical enquiry in the scholarship of learning and teaching. Our findings provide initial insights, but more work is required to examine the benefits and limitations of an AI approach across higher education settings internationally. Finally, through detailing how organizations can foster appreciative and affirmative practices of PR, our paper contributes tangible recommendations for undertaking PRs that accord with the principles of AI. Our experiences indicate that treating peer review as an exercise in AI, rather than as a performance-based measurement exercise, can improve workplace practices through increasing collaboration, mutual support and collegiality. Fostering such collaboration, support and collegiality in higher education is particularly important given that universities are increasingly reflecting neoliberal values such as individualisation, privatisation and the endless pursuit of 'efficiencies' (Connell, 2019). Expanding peer review as an exercise in AI could help resist some of the pressures and constraints of the austerity university (Giannacopoulos, 2019), however, we want to stress that a robust, sustainable program of peer review as AI requires a broader ecological shift away from PR's longstanding association with audit culture. Given that the relations we enter into "shape what we are able to produce" (Giannacopoulos, 2019), how we support each other in producing better teaching and learning practices, can contribute to a more collegial environment.

Moreover, it is not just the outcome of peer review that is important: we must also value how we conduct peer review and the sorts of relations it generates, both for teachers and students. From the outset, we approached PR as a process that can have clear implications for learning. Any changes to teaching practice are only as beneficial as they might be to student learning since teaching and learning are constitutive of each other. At each stage of the process we were mindful of reviews which would lead to more engaged and engaging learning for students. This consideration is important within an Australian context as well as internationally, given the inter-relationship between teaching quality and student retention and engagement (Crosling, Heagney \& Thomas, 2009). As Crosling and colleagues (2009: 9) observe, retention and learning outcomes represent "an issue of concern in higher education institutions across the world", with follow on implications for the financial and reputational well-being of academic institutions - particularly within hyper-competitive global markets - as well as for society more broadly "through the loss of potential skills and knowledge" (see also Burke, 2019). While the factors underpinning student retention are complex and multi-faceted, the quality of teaching and learning plays some role (Crosling, Heagney \& Thomas, 2009). In developing an innovative model of PR that is generative rather than evaluative or punitive in approach, we suggest that an AI approach to PR has the potential to cultivate 'best practice' teaching, and contribute to enhancing student retention, engagement and learning outcomes.

In order to develop our argument, we begin by examining relevant literature on PR, highlighting dominant conceptual approaches. We then detail AI, and its use in other fields, including higher education, providing context for findings from our reflections on undertaking reciprocal, formative reviews of each other's teaching practice. While we initially approached the PR process with a sense of anxiety and apprehension, in practice we found that the review process functioned to identify and build upon what we were doing 'well' as educators. Based on our findings, we then detail how PR can be approached as a form of AI, and mount a normative argument for the potential benefits of approaching PR in this way.

\section{Literature Review}

\section{The nature and challenges of peer reviews of teaching}


Multiple studies have detailed how educators in diverse disciplines can benefit from PRs of teaching (Prezas et al., 2009). PRs represent a valued source of feedback on teaching practices (Hatzipanagos \& Lygo-Baker 2006), particularly relating to teacher immediacy: how teachers' communication can reduce psychological and/or physical distance between teachers and students, for example (Nixon et al., 2010; see Christophel \& Gorham 1995). Further, effective PRs of teaching can generate benefits both for the reviewer and the reviewee. As Golparian et al. (2015, p. 212) note, a PR of teaching "provides an opportunity for the reviewee to have feedback on their teaching, and it exposes the reviewer to different styles and techniques of teaching". PR can also work to strengthen collegial relationships, in addition to enhancing pedagogical and professional development (Chester et al., 2013). However, these benefits are not reported in all studies (Blackmore, 2005). Potential limitations to PR include reviewers' reluctance to give their colleagues negative feedback (Blackmore, 2005; Iqbal, 2014; Yiend et al., 2014), and that PRs often examine only a limited range of presentation-related issues (Crutchley et al., 2005). In some cases, PR of teaching schemes face obstacles to securing faculty 'buy-in', with many staff (often rightly) associating PR purely with evaluation and compliance (Shortland, 2004).

Given these limitations, it is perhaps unsurprising that much of the literature on PR of teaching has examined the conditions supporting successful PR (Iqbal, 2014). This body of work has identified a range of factors associated with more successful reviews. However, what constitutes 'success' in peer review will likely differ depending upon the purpose and aims of the review, and the subjective experience(s) and interpretation(s) of the individuals involved in the review process. Indeed, it is important to qualify that numerous models of PR exist, often serving very different purposes. PRs of teaching can fall along a continuum of (in)formality (Golparian et al, 2015), with formal or summative PRs associated with teaching evaluation and compliance as opposed to professional development. Informal or formative PRs, by contrast, are employed primarily for the purpose of professional development.

Further, Cassidy and Johnson (2006) note that the style of feedback provided to reviewees is one factor shaping review success. In light of this, Cassidy and Johnson (2006), emphasize the importance of constructive dialogue between reviewer and reviewees, something also reflected in Gosling's (2014, pp. 22-23) application of Habermasian principles of communicative understanding to collaborative PR (see Habermas, 1984). Additionally, Farrell (2011, p. 6) draws attention to the importance of recognizing that "peer review will only work well if colleagues respectfully acknowledge that there are diverse approaches to teaching", with the reviewer helping to promote critical reflection and "strategies to address any areas for improvement". Together, this indicates the importance of allowing for flexibility and fluidity in the approach taken to PR, and in understanding what constitutes 'good' or 'effective' teaching practice.

The uptake of PRs of teaching within higher education is also shaped by departmental culture, including the value placed on collegiality, which can substantially shape colleagues' feedback practices (Iqbal, 2014). Yet within some faculty cultures, even providing constructive feedback can be perceived as "divisive, difficult, and a violation of norms" (Iqbal, 2014, p. 117). Strategies such as ensuring that reviewers' reports remain confidential to reviewees (Cassidy \& Lee, 2011) and modelling effective critical feedback behaviors (Yiend et al., 2014) may help to mitigate these challenges. Similarly, there is a need to explain the precise purpose of a PR scheme (Cosh, 1998; Hammersley-Fletcher \& Orsmond, 2004) through, for example, running introductory workshops explaining the principles of effective reviewing (Cassidy \& Lee, 2011; Golparian et al., 2015). Nonetheless, the unique cultural and social 
contexts of institutions may pose challenges to the successful implementation of PR. As we argue, AI may present another strategy for circumventing such challenges.

\section{Appreciative inquiry}

Originally developed by Cooperrider and Srivastva (1987), AI is a strengths-based approach to generating change (Bushe, 2013). Originating in organizational design and change, AI has since been applied to a range of other settings, including higher education. AI is based on the notion that by focusing on the negatives or deficits of a particular organization, "we simply create more images of deficit and potentially overwhelm the system with images of what is "wrong" (Watkins, Mohr \& Kelly, 2011, p. 16, emphasis added; see also Giles \& Kung, 2010; Harrison \& Hasan, 2013). In contrast, AI asks us to "have an image of how that situation ought to be", directing our focus and energy towards this ideal vision (Watkins, Mohr \& Kelly, 2011, p. 16; see also Giles \& Kung, 2010; Harrison \& Hasan, 2013; Kung et al., 2013; Scott \& Armstrong, 2019). In this sense, AI is both generative and forward-looking, honing our attention on where we want to be and how we want to be. Additionally, AI can help to identify what we already do 'well' in our professional practice, bringing attention to what we should continue or 'do more of' (De Lautour, 2009; Johnson, 2014).

In practice, the AI methodology is underpinned by a four-phase process, commonly referred to as the 4D model or the 4Ds of AI (Mohr \& Watkins, 2001; Watkins, Mohr \& Kelly, 2011). De Lautour (2009, p. 81) summarises the 4Ds of AI as:

- Discovery: all members are part of the process of identifying the strengths and positive aspects of current practice;

- Dream: envisioning and conceiving the possibilities for the key questions that arise from the previous process or phase;

- $\quad$ Design: developing 'propositions' (structured goals) that appear possible and attainable; and

- Destiny: realizing and strengthening of these propositions in a positive and fulfilling manner.

As Giles and Kung (2010, p. 309) attest, AI's 4D model provides a framework for shifting away from deficit-thinking, as a deficit approach constructs our worlds in a way that "lead[s] to a greater sense of hopelessness". Drawing on post-structuralist approaches, AI's shift away from deficit thinking allows us to (co)construct 'best practice' in pedagogy - it is a practice of discursively constituting the world (Harrison \& Hasan, 2013; Johnson, 2014). Viewed from an AI perspective, to achieve 'best practice' in teaching, we need to make elements of our pedagogical practices visible without fomenting hopelessness. Consonant with the AI approach, Harrison and Hasan (2013, p. 67) suggest, for example, that "when students and educators are bombarded with incomplete, negatively slanted representations of themselves, they internalize them". Our identity as educators is formed (at least in part) through our social relationships - our interactions with and feedback received from peers plays a central role in constructing our reality (Johnson, 2014). In other words, a deficit-approach to PR and teaching evaluation creates a context in which negative elements of our teaching practice are amplified, with subsequent implications for our sense of identity and capacity as educators.

Despite the widespread application of AI in other fields, and to a lesser extent other facets of higher education (Johnson, 2014; Jones \& Masika, 2020; Kadi- Hanifi et al., 2014; Kung et al., 2013; Lane et al., 2018), there is little development of this framework in relation to PR and none in higher education. Though Van der Vaart's (2017) has developed a procedure for 
undertaking an "Appreciative Peer Review", their PR procedure was designed for an organizational rather than higher education setting specifically. Elsewhere, Giles and Kung (2010, p. 311) reflect on using AI in identifying examples of "peak professional practice" in higher education. For Giles and Kung, this involved working with a colleague to generate examples of 'best practice' teaching encounters and using these to generate actions that allow the teacher to "experience further life-giving moments of professional practice" (2010, p. 318). Thus, while Giles and Kung's (2010) work examines the application of AI in higher education, AI is not examined in the context of 'traditional' PR. Harrison and Hasan (2013, p. 69) advocate for the use of AI in transforming relationships between students and teachers in higher education away from one of "opposition" or defensiveness towards "quality learning environments" (see also Johnson, 2014; Kung et al., 2013).

Reflecting on her experiences applying an AI framework to tertiary teaching, De Lautour (2009) found that AI aligned with and supported her teaching philosophy and approach to professional development, namely of "'growing' in the direction of that which is positive, fulfilling and potentially leads to greater connections" (2009, p. 81). In contrast, De Lautour suggests that the narrow criteria set for teaching review in her own institution worked against the open - and largely positive - feedback she received through asking students to provide anonymous handwritten feedback. In De Lautour's case, AI was used in the context of student feedback and a collaborative research project, rather than PR, though these processes arguably share many similarities.

Giles and Kung suggest that the AI process in higher education "requires positive affect and a relational connection to a colleague or critical friend" (2010, p. 311). Similarly, Harrison and Hasan (2013, p. 72) argue that institutional context "plays a critical role in determining whether a deficit or AI paradigm will prevail...[and] can enhance or inhibit an AI perspective". This institutional context includes external pressures, metrics and regulatory frameworks, which tend to focus on a deficit model (Scott \& Armstrong, 2019). Undoubtedly, such pressures apply to the university sector, with quantitative metrics of 'success' taking primacy within neoliberal higher education institutions (see Connell, 2019). Within such 'entrepreneurial universities' (Slaughter \& Leslie, 1997), generalised competition represents a behavioural imperative informing staff research (Craig et al., 2014), engagement (Richards et al., 2020), and teaching (Shore and Wright, 2003) through the tools of audit culture (Dardot and Laval 2014). As a result, the broader context of the neoliberal university may curtail the possibilities for employing AI in PR. The benefits of PR when undertaken within the framework of AI, however, show how PR can deliver much more meaningful benefits beyond those produced when the focus is metrically driven.

\section{Process \& Method}

In the remainder of the article, we draw on our reflections from undertaking reciprocal, formative PRs of each other's teaching practice. These reviews were undertaken as part of the requirements of the Graduate Certificate in University Teaching, which Bianca was enrolled in at the University of Melbourne. Completing the compulsory subject Teaching in Practice required her to conduct two reciprocal PRs, and to observe the teaching of two colleagues within her own discipline. The PRs and observational notes formed the basis for a teaching portfolio to be submitted as an assessment task, as well as informing a final reflective essay. The PRs and observations were otherwise formative in nature; they were not attached to any formal institutional processes of compliance or performance review. 
As part of this process, Bianca and Claire, and Mark and Bianca, engaged in reciprocal PRs. Our reviews occurred across a range of different teaching contexts within the Criminology discipline as follows:

- Bianca and Claire were co-teaching a large ( 700+), introductory first year Criminology subject, and reviewed each other's lecture delivery in this course. This subject is a flagship Criminology subject which takes students through a series of case studies and theoretical approaches to the study of crime.

- Bianca reviewed Mark's lecture delivery for a mid-sized $(\sim 150+)$, $3^{\text {rd }}$ year undergraduate subject. In turn, Mark reviewed Bianca's delivery of a guest seminar in a post-graduate research methods subject ( $\sim 30$ students).

Before each review the reviewee completed a form providing background information about the subject (e.g., level and number of students), the aims of the session being observed, and any areas of concern they wanted reviewer feedback on. Each reviewer observed the entire teaching session, taking notes throughout. After the session, the reviewer compiled a written report for the reviewee, using an institutional template. While the template contained a section for the reviewee to respond to the reviewer's feedback, this opportunity was not taken up in any of the reviews (though, as we outline later, this may be necessary for PR to 'truly' function as $\mathrm{AI})$.

As two of the subjects were large and held in cavernous lecture theatres, the presence of the peer reviewer was not announced to (or, apparently, noticed by) students. In the smaller postgraduate session, Bianca had been invited to deliver a guest seminar, and Mark was the primary seminar leader and subject co-ordinator, so students were not informed that a review was being conducted. Our PRs thus spanned a range of different teaching contexts within our discipline. Clearly, the insights garnered here arise from a small number of reviews and the reflections of a small number of educators. Whilst we do not claim that our insights are generalizable, they do point towards innovative ways of conceptualizing and implementing PR, and new avenues for pedagogical inquiry.

We emphasise that the value of an AI approach to PR emerged organically as an effect of our reflections on the peer review process. We found this illuminating and were keen to examine how this could be retroactively mapped to the first two stages of the AI process. Accordingly, we limited our analysis to the first two stages of AI, that of "Discovery" and "Dream", showing how we were able to "discover" the strengths in our teaching practices, and to "dream" about transformative possibilities. We argue that this preliminary work opens up a space for applying the final two stages of AI to PR of teaching - that of "Design" and "Destiny" - in future PRs of teaching practices.

\section{Findings: Peer Reflections on Peer Review Peer review as Appreciative Inquiry}

Like many who undertake PR, we experienced a level of anxiety in the lead up to having our teaching reviewed. Bianca, for example, was concerned that her reviews would be negative, drawing attention to what she was doing 'wrong' in her teaching practice. As an early-career lecturer on a three-year contract, Mark worried that negative assessments of his teaching - even outside a formal evaluation setting - may have implications for his employability. And while Claire had extensive teaching experience for an early career academic, she was concerned that she did not convey material in sufficient depth, given her focus on getting students to absorb key messages through active engagement in the classroom. At the same time, and 
paradoxically, she was concerned about the heavy amount of text on her PowerPoint slides. Such affective states are likely driven by a sense of 'imposter syndrome' - anecdotally rife in academic settings - where we feel that we are not competent at our work and anxious about a lack of skill being exposed (see Breeze, 2018). These anxieties are likely also underpinned by reviewees associating PR with summative evaluations of our teaching, with potential consequences for not performing 'well' enough.

However, in practice, we found that our reviews instead reflected the 'Discovery' phase of AI. That is, they largely worked to elucidate what we 'did well' in our teaching, as illustrated through the following comments:

The clarity, succinctness and detail of the lecture was admirable. Bianca covered a wide range of ideas, research and examples but did so in a way that never felt compressed. The slides were pleasurable to read, and communicated content in a systematic way. (Claire review of Bianca)

I thought your discussion of theory was clear and effective, you explained key theories in detail and/or referred back to what had been addressed in previous lectures to refresh students' memories. You effectively used examples to make theoretical concepts more tangible (Bianca review of Mark)

In line with AI, these reviews functioned to identify what we should do 'more of' in our teaching. As indicated earlier, this process can be understood as actively constructing or producing 'effective' teaching practice. In this respect, AI can be viewed as fundamental to developing best practice pedagogy, as opposed to being mindlessly positive at the expense of critique. In Claire's case, some of the feedback also helped to highlight what was working well, exemplified in comments like:

Claire consistently demonstrated a range of effective communication techniques [including] clear signposting of session content... how the lecture 'fits' within the broader context of the course, and how it builds on content that has been covered so far... [through] explicit emphasis of key points. (Bianca review of Claire)

This feedback affirmed her approach to first year, undergraduate teaching, in which she consciously adopts a scaffolded learning approach, aiming to show connections across diverse subject materials.

While our reviews tended to emphasize positive aspects of each other's teaching, we nonetheless still identified areas for improvement as the following comments illustrate:

Some slides had very large amounts of written text that wasn't...discussed. I did wonder if some of the slides could be streamlined or reduced to avoid cognitive overload and students potentially feeling overwhelmed? (Bianca review of Claire)

Claire was not surprised by this comment, as she had been aware of her tendency to over-rely on text. In part, this was a technique she reported using to avoid reading lecture notes, with the slides providing prompts for lecture delivery to enhance an informal teaching mode. These comments illustrate that AI does not necessarily mean that critical comments are purposefully avoided - some critique may even be necessary to shift towards 
the 'dreaming' and 'design' phases of AI, as we will describe below. Whether AI should include critique and the identification of challenges or weaknesses that need to be overcome is a point of considerable contention in the literature. Some AI scholars maintain that the process should focus on the unconditionally positive (Kadi-Hanifi et al., 2014). In contrast, others, such as Bushe (2007), place emphasis on being generative rather than unceasingly positive. Jones and Masika (2020, p.4) similarly argued that it was necessary to identify challenges faced by first-year students in their study, and that applying AI in this way "provides students from diverse backgrounds with greater voice and agency that can help contribute towards positive change". In this way, AI points towards where we would like to progress to in our practice, rather than points to dwell on or respond to. Importantly, it also suggests the value of AI-guided PR in responding to student need and therefore to potentially contribute to student retention.

Moreover, we found that PR functioned as a form of AI in a reciprocal and collective manner. This suggests the possibility of collaboration between colleagues, instead of evaluative judgements entailing a "power relationship" (Gosling 2014, p. 17). As noted, much of the literature in relation to PR focuses on the benefit of peer review to the reviewee, and in particular the benefit for junior scholars, and to the quality of teaching in higher education (Lomas \& Nicholls, 2005; Thomas et al., 2014). However, we argue, as have others (Gosling, 2014; Peel, 2005), that the benefits of PR extend far beyond this. PR also offers reviewers (or observers) an opportunity to reflect on their own practices, feeding into best practice teaching for all parties conducting the review (see also Golparian et al., 2015).

One common assumption is that PR is undertaken by colleagues who are senior to the person being reviewed, and who are often considered teaching and/or disciplinary experts (Georgiou et al., 2018). Here, PR is seen as a "top-down, rather than a bottom-up process" (Peel, 2005, p. 492). Naturally this raises questions about what is meant by the reference to 'peer' in PR for example, it is questionable to what extent senior academics are actually the 'peers' (a term which implies a level of equality or equal standing) to their early career counterparts. But it also overlooks the value of junior colleagues reviewing the performance of those senior to them. Seeing other teachers in their own practice can make visible not just what but how others teach. For early career academics, this may be particularly important. For us, PR was a reciprocal and mutually beneficial process. Undertaking PR provided a pedagogical function for the reviewer - it helped us to learn about 'effective teaching' through observation and critical reflection. This process of reciprocal PR and observing colleagues' teaching practices reflects both the 'discovery' and 'dreaming' phases of AI. Observing one's peers may help us to identify or 'discover' elements of competent teaching practice. Additionally, in learning about the diverse and (potentially) innovative practices of other educators, PR may allow us to envisage and conceive of the possibilities for developing our own teaching approach, reflecting the 'dreaming' phase of AI (DeLautour, 2009).

For example, Claire recounted her early tutoring experiences, where, due to limited training and support at that time,,she felt that she had 'no idea' about what what constituted effective teaching strategies, and lacked clear strategies for dealing with challenging class dynamics. In the absence of a formal PR process, she found that it was the casual or incidental opportunities she had to review and observe others' teaching practices that enabled her to develop her own teaching strategies and adapt them to her own personal style. For Claire, this affirmed the value of observation, as well as that of being observed. However, more was required than simple observation. Reflective practice was also critical, something which was not enforced through a performance appraisal system, but which she chose to adopt as part of her own professional learning, as "a habit of mind" (Gosling, 2014, p. 27). There is also a tendency to assume, 
including in AI literature, that learning and improvement occurs through feedback from reviewer to reviewee in a formal process. But there are many, less formal examples where we can learn from others through active exposure to the teaching practices and methods of more experienced teachers, including those with diverse teaching styles. We learn by watching and reflecting on the work of others, as well as by being reviewed and observed ourselves. Claire valued this as the 'accidental benefit' of her informal observation of the teaching of others, the benefits of which would potentially be undermined by a metrically-driven review process. Indeed, the latter can obscure the subtle ways in which we learn from each other, which are not always reducible to a process-driven, evaluative approach.

As our own experience involved only one cycle of reciprocal review, we did not neatly capture the design and destiny phases of AI. However, we can see how these elements of AI translate across to the PR process. For instance, the 'design' phase of AI embeds a process of reflection and strategies for change in $\mathrm{PR}$ - a practice that is already emphasized in pedagogical literature (Biggs \& Tang, 2011). This phase of AI asks what we plan to do in response to the insights generated through the discovery and dreaming phases. In other words, what is the road map that will take us towards doing more of what we do 'well' in our teaching? Finally, the 'destiny' phase of AI involves "realizing and strengthening of these propositions" (De Lautour, 2009, p. 81). In relation to teaching, this could be thought of as the implementation and review phase: the point at which we introduce tangible changes to our practice and seek to evaluate and refine the outcomes.

It is also important to reflect on why and how our experience of PR was able to function as AI. This was not necessarily a pregiven outcome of the PR process - as detailed earlier, the institutional context plays a central role in shaping how PR is implemented and for what purpose (Harrison \& Hasan, 2013; Scott \& Armstrong, 2019). Rather, we argue that several contextual and relational factors shaped this outcome. Firstly, the PR tools developed within our institution lent themselves to an AI-based approach. Whilst some other applications of AI in university settings, such as student reviews of teaching (De Lautour, 2009), may struggle to generate the conditions required for the approach to operate effectively, in our own case, the relatively open-ended design of the PR forms in fact did allow for feedback that reflected principles of AI. For example, rather than constituting a quantitative and/or evaluative tick box exercise, our institutional template consisted of qualitative and open-ended reviewer comments, giving us scope to emphasize in detail examples of best practice. The danger of focusing centrally on what needs improvement is made clear here: knowing what works well is often as critical to best practice as addressing areas which require change. It can help educators to harness the positive aspects of their teaching and to refine them. This suggests that institutional processes and the design of review instruments may be central in producing reviews that align with AI.

Additionally, the nature of our relationships as colleagues also contributed to PR functioning as AI. Collegial relationships are central to the outcomes of both PR and AI (Giles \& Kung, 2010). There is a considerable amount of vulnerability generated in the processes of PR and AI, so approaching PR as AI likely requires substantive levels of trust and collegiality that may not exist in some higher educational contexts (Iqbal, 2014). In our case, our collective status as early career researchers flattened out potential power differentials or hierarchies. Although Claire is a more experienced colleague in terms of her teaching history, all participants enjoy a similar status as early career researchers and are employed at similar levels. We approached the reviews as relative equals, and this perhaps reduced the perceived threat of negative consequence or of being judged by a 'superior' (even if the experience was still a daunting 
one!). This is not to say that colleagues in differential positions of power or levels of experience cannot undertake PR as a form of AI, only that it appeared to facilitate it to function in this way in our own experience. Likewise, as our reviews were reciprocal in nature, this likely also shaped our tendency to given more positive feedback.

\section{Discussion: Fostering peer reviews of teaching as appreciative inquiry}

Numerous studies have indicated the utility of an AI approach to instigating change within organizations, including higher education institutions (Allen \& Innes, 2013; Collington \& Fook, 2016; Fifold \& Stowe, 2011; Fifolt \& Lander, 2013). For this reason, we suggest that reframing PRs of teaching through an explicitly AI approach may offer one vehicle for normalizing such reviews as a practice within higher education institutions. Through shifting the frame from an analysis of pedagogical problems to a dialogue envisioning 'what might be' and 'what could be' in pedagogical practice, adopting an AI framework for PR of teaching may, we suggest, reduce feelings of tension and appraisal fatigue among faculty. It might even be enjoyable.

One key step to normalizing PR is addressing some of the concerns staff may have around PRs of teaching (Lomas and Nichols, 2005). As we have discussed, one of these key concerns is that it represents a form of appraisal, as opposed to a professional development practice. PR, in short, can readily be reduced to a form of quality assurance, ensuring that teachers meet externally-imposed standards. This can be exacerbated when reviewers and reviewees are of different status within a department or discipline (Kinchin, 2005). To date, scholars have identified two key ways we might address this concern, both of which are consonant with (re)framing PRs of teaching as AI: framing PR as professional development and emphasising the benefits to both reviewees and reviewers. In detailing each of these approaches, we discuss how the insights of AI may be explicitly drawn upon to overcome common barriers to a culture of PR in higher education.

Firstly, we can change staff perceptions of PR through explicitly aligning it with quality enhancement and professional development. Another way of articulating this is through approaching the PR of teaching as a formative rather than a summative process (Bingham \& Ottewill, 2001). Rather than placing PR of teaching alongside other mechanisms for assessing the effectiveness of our teaching practice, we might instead place PR among the cornucopia of professional development exercises we might employ to become more effective educators. Given that PR is a central aspect of our research activities, it makes sense to embed a collegial PR process in our teaching to support each other in achieving best practice. In line with the principles of formative assessment, we might understand PR of teaching as essentially positive in intent; a means of promoting new forms of teaching practice, particularly in the case of reciprocal reviews (Harlen \& James, 1997). This understanding of PR as essentially positive in intent is strongly redolent of AI, which, as we have detailed, promotes organizational change and development through harnessing the power of positive emotions, dialogue and images of desired futures (Watkins et al., 2011). Reframed as AI, PRs generate affirmative images of each other's teaching and, in doing so, generate new pedagogical ideas, images and practices to bring into the seminar room and the lecture theatre.

AI's strengths-based approach provides an excellent framework for emphasizing how PR may benefit both the reviewer and the reviewee (Lomas \& Nicholls, 2005). This extends the notion of collaborative PR identified by Gosling (2014) by showing how the principles he identifies (reciprocal learning, enquiry, and a non-judgemental approach) might be realized in practice, through AI principles. Namely, an AI approach facilitates the ability for peer reviewers to 
apply new pedagogical strategies in their own teaching practice, through identifying what works well in others' teaching practices. This key benefit to peer reviewers is downplayed when we view PR as primarily about identifying and resolving problems in the teaching practices of others. An AI-informed PR foregrounds what is best in others' teaching, and asks questions that seek to understand, anticipate and increase the positive potential in others and our own teaching. Through the reflective component of AI, we can carefully consider the teaching style that might work best for us, as individuals. This recognizes that there are many different ways to teach, rather than assuming that there is a single, best way to teach which must comply with a formulaic pedagogical model.

The dream and design phases characterising AI are typically absent from more appraisallike PR but can be used to overcome such a formulaic approach. In the dream phase of an AI approach, reciprocal peer reviewers/reviewees share images and experiences of exceptional teaching. In doing so, they address a series of crucial questions: what might exceptional teaching at their institution look like? What would the conditions be for such exceptional teaching to become the norm, rather than the exception at their institution (see Watkins et al., 2010)? In addressing these questions, peer reviewers develop guiding principles for fostering the best aspects of their teaching, drawing on examples of good practice identified in the Discovery phase of the PR. In the Design phase of an AI-informed review of teaching, reciprocal peer reviewers/reviewees then develop these principles to generate plans for pedagogical change so that the conditions for fulfilling the dream phase are enabled.

As Collington and Fook (2016) suggest, for AI to engender positive change within higher education, staff must be keenly aware of the approach's underlying principles. Like Collington and Fook (2016), we suggest that faculties considering applying AI principles in implementing PR of teaching programs devote time and energy to the process. This would include delivering introductory sessions that set out the principles underpinning a distinctly AIinformed approach to PR. Such introductory sessions are key to any AI project, wherein facilitators may explain the inquiry's goals, protocol, and framing questions. In the case of AIinformed PR of teaching, introductory sessions provide a platform for detailing the purpose and value of an AI-informed PR, how it will be undertaken and how it might contribute to reflective practice.

\section{Conclusion}

In this article, we have mounted a two-fold argument for reframing PR as AI. Firstly, our findings provide empirical insight into how PR can be undertaken as a form of AI in a way that may overcome some of the challenges traditionally associated with PR. Secondly, we have mounted a normative argument outlining the potential benefits of undertaking PR using an AI approach. Based on our findings, we have argued that when PRs of teaching are reframed as AI, PR becomes a generative rather than evaluative exercise. Specifically, AIinflected PR generates an understanding of new ways to develop our teaching. Drawing on AI's principles, PR of teaching can be used to amplify the best aspects of one another's teaching and bring the best aspects of other's teaching into our own. Undertaking PR as an exercise in AI thus supports two-way learning and can help advance our teaching. While sometimes well aware of our own limits, when AI is embedded in PR, we can be better equipped to make changes which produce a substantive difference in how we teach as well as become more confident in our own teaching style. The risk of reducing PR to a metricallydriven, performance appraisal exercise is that it could also encourage a standardised approach to teaching which then deprives students of exposure to high quality, diverse teaching practices. Moreover, PR can be a transformative process which shapes our identities as teachers (Pell, 
2005). We thus suggest that when PR is undertaken using the principles of AI, it is better equipped for "enabling change for the better" in developing "one's professional craft" which is closely tied to how we see ourselves as teachers (Peel, 2005, p. 489). The very term 'review' carries the baggage of evaluation - baggage, we might suggest, that might fruitfully be deposited in favor of a more accurate descriptor: appreciative peer inquiries of teaching. These empirical insights into, and innovative conceptualization of, PR has the potential to shift how PR is done and for what purpose. We believe this approach may in turn enhance the quality of teaching in higher education, with potential follow-on ramifications for student learning, engagement and retention. While these claims of course require further empirical testing and exploration - and the relationship between appreciative peer inquiries of teaching and these outcomes is likely complex, fluid, and far from certain - we offer this conceptual and practical intervention in the hope of initiating a journey of generative change.

\section{Declaration of interests}

The authors have no conflict of interest to declare.

\section{References}

Allen, J. M., \& Innes, M. (2013). Using appreciative inquiry to frame the appraisal of an Australian initial teacher education program. Australian Journal of Teacher Education, 38(11), 1-17.

Bell, A., \& Mladenovic, R. (2008). The benefits of peer observation of teaching for tutor development. Higher Education, 55, 735 - 752.

Bingham, R., \& Ottewill, R. (2001). Whatever happened to peer review? Revitalizing the contribution of tutors to course evaluation. Quality Assurance in Education, 9(1), 3239.

Blackmore, J. A. (2005). A critical evaluation of peer review via teaching observation within higher education. International Journal of Educational Management, 19(3), 218-232.

Breeze, M. (2018). Imposter syndrome as a public feeling. In Y. Taylor \& K. Lahad (Eds.). Feeling academic in the neoliberal university: Feminist flights, fights and failures (pp. 191-219). London, UK: Palgrave Macmillan.

Burke, A. (2019). Student retention models in higher education: a literature review. College and University, 94(2), 12-21.

Bushe, G. (2007). Appreciative inquiry is not (just) about the positive. $O D$ practitioner, 39(4), 30-35.

Bushe, G.R. (2013). The Appreciative Inquiry Model. In E. Kessler (ed.) The Encyclopedia of Management Theory. London UK: SAGE Publications.

Cassidy, A. \& Johnson, J. (2006). Developing your skills as a peer reviewer: Introductory workshop handbook. Centre for Teaching and Academic Growth. University of British Columbia.

Available at: https://wiki.ubc.ca/Documentation:CTLT_programs/PRT/UBC_docs 
Cassidy, A., \& Lee, J. $\quad$ (2011). Peer review: Structured, informal, confidential, helpful! Collected Essays on Learning and Teaching, 4, 68-73.

Chester, A., Clarke A., Wingrove, D., \& Denny, B. (2013). Talking about teaching: empowering staff through peer partnerships. Proceedings of the $10^{\text {th }}$ Enhancement and Innovation in Higher Education conference. Edinburgh

Christophel, D. M., \& Gorham, J. (1995). A test-retest analysis of student motivation, teacher immediacy, and perceived sources of motivation and demotivation in college classes. Communication Education, 44(4), 292-306.

Collington, V., \& Fook, J. (2016). Instigating change through Appreciative Inquiry: A case study. International Journal of Higher Education Management, 3(1), 1-13.

Connell, R. (2019). The Good University: What Universities Actually Do and Why Its Time for Radical Change. London, UK: Zed Books Ltd.

Cooperrider, D., \& Srivastva, S. (1987). Appreciative Inquiry in organizational life. In R.W. Woodman \& W.A. Pasmore (eds.). Research in Organizational Change and Development (pp. 129-169). Vol.1. Stamford, US: JAI Press.

Cosh, J. (1998). Peer observation in higher education - A reflective approach. Innovations in Education and Training International, 35(2), 171-176.

Craig, R., Amernic, J., \& Tourish, D. (2014). Perverse audit culture and accountability of the modern public university. Financial Accountability \& Management, 30(1), 1-24.

Crosling, G., Heagney, M., \& Thomas, L. (2009). Improving student retention in higher education: improving teaching and learning. Australian Universities' Review, 51(2), 9-18.

Crutchley, D., Nield, K., \& Jordan, F. (2005). Moving on from peer observation of teaching: A collaborative development using the principle of peer-support. Educational Developments, 6(1), 1-5.

Dardot, P., \& Laval, C. (2014). The new way of the world: On neoliberal society. London, UK: Verso.

De Lautour, N. (2009). Through others' eyes: Reflections of a tertiary teacher. New Zealand Journal of Teachers' Work, 6(1), 79-89.

Farrell, K. (2011). Collegial feedback on teaching: A guide to peer review. University of Melbourne Centre for the Study of Higher Education.

Fifolt, M., \& Lander, L. (2013). Cultivating change using appreciative inquiry. New Directions for Student Services, 143, 19-30.

Fifolt, M., \& Stowe, A. (2011). Playing to your strengths: Appreciative inquiry in the visioning process. College and University, 87(1), 37-40. 
Georgiou, H., Sharma, M., \& Ling, A. (2018). Peer review of teaching: What features matter? A case study within STEM faculties. Innovations in Education and Teaching International, 55(2), 190-200.

Giannacopoulos, M. (2019). 'Nomophilia and Academic Work in the Austerity University'. Journal of Global Indigeneity, 4(1).

Giles, D., \& Kung, S. (2010). Using appreciative inquiry to explore the professional practice of a lecturer in higher education: Moving towards life-centric practice. Australian Journal of Adult Learning, 50(2), 308-322.

Golparian, S., Chan, J., \& Cassidy, A. (2015). Peer review of teaching: Sharing best practices. Collected Essays on Learning and Teaching, 8, 211-218.

Gosling, D. (2014). Collaborative peer-supported review of teaching. In J. Sachs \& M. Parsell (eds) Peer Review of Learning and Teaching in Higher Education (pp. 13 - 31). Springer.

Habermas, J. (1984) The theory of communicative action: Reason and the rationalization of society (Vol I), Boston, MA: Beacon Press.

Hammersley-Fletcher, L., \& Orsmond, P. (2004). Evaluating our peers: is peer observation a meaningful process? Studies in Higher Education, 29(4), 489-503.

Harrison, L. M., \& Hasan, S. (2013). Appreciative inquiry in teaching and learning. New Directions for Student Services, 143, 65-75.

Hatzipanagos, S., \& Lygo-Baker, S. (2006). Teaching observations: promoting development through critical reflection. Journal of Further and Higher Education, 30(4), 421-431.

Iqbal, I. A. (2014). Don't tell it like it is: Preserving collegiality in the summative peer review of teaching. Canadian Journal of Higher Education, 44(1), 108-124.

Johnson, B. A. (2014). Transformation of online teaching practices through implementation of appreciative inquiry. Online Learning, 18(3), n3.

Jones, J., \& Masika, R. (2020). Appreciative inquiry as a developmental research approach for higher education pedagogy: space for the shadow. Higher Education Research \& Development, advance online publication.

Kadi-Hanifi, K., Dagman, O., Peters, J., Snell, E., Tutton, C., and Wright, T. (2014). Engaging students and staff with educational development through appreciative inquiry. Innovations in Education and Teaching International, 51(6), 584-594.

Kinchin, I. M. (2005, September). Evolving diversity within a model of peer observation at a UK university. In British Educational Research Association (BERA) Annual Conference, University of Glamorgan, Wales (pp. 14-17). 
Kung, S., Giles, D., \& Hagan, B. (2013). Applying an Appreciative Inquiry Process to a Course Evaluation in Higher Education. International Journal of Teaching and Learning in Higher Education, 25(1), 29-37.

Lane, K., Teng, M. Y., Barnes, S. J., Moore, K., Smith, K., \& Lee, M. (2018). Using Appreciative Inquiry to Understand the Role of Teaching Practices in Student Wellbeing at a Research-Intensive University. The Canadian Journal for the Scholarship of Teaching and Learning, 9(2), 10.

Lomas, L., \& Nicholls, G. (2005). Enhancing teaching quality through peer review of teaching. Quality in Higher Education, 11(2), 137-149.

Mohr, B.J., \& Watkins, J.M. (2001). Resource book for appreciative inquiry: A constructive approach to organizational development. Bethel, ME: NTL Institute.

Nixon, S., Vickerman, P., \& Maynard, C. (2010). Teacher immediacy: Reflections on a peer review of teaching scheme. Journal of Further and Higher Education, 34(4), 491502.

Peel, D. (2005). Peer observation as a transformatory tool? Teaching in Higher Education, 10(4), 489 - 504.

Porter, C., Moore, C.M., Boss, G.J., Davis, T., \& Louis, D.A. (2020). To be Black Women and contingent faculty: Four scholarly personal narratives. The Journal of Higher Education. Advance online publication.

Prezas, R., Shaver, M., Carlson, T., Taylor, J. S., \& Scudder, R. (2009). Peer review of teaching: Multiple raters. Perspectives on Issues in Higher Education, 12(2), 59-63.

Richards, I., Wood, M. A., \& Iliadis, M. (2020). Newsmaking criminology in the twenty-first century: an analysis of criminologists' news media engagement in seven countries. Current Issues in Criminal Justice, 32(2), 125-145.

Scott, J. T., \& Armstrong, A. C. (2019). Disrupting the deficit discourse: reframing metaphors for professional learning in the context of appreciative inquiry. Professional Development in Education, 45(1), 114-124.

Shore, C., \& Wright, S. (2003). Coercive accountability: the rise of audit culture in higher education. In Strathern, M. (Ed.) Audit cultures. London, UK: Routledge. pp.69-101.

Shortland, S. (2004). Peer observation: A tool for staff development or compliance? Journal of Further and Higher Education, 28(2), 219-228.

Slaughter, S., \& Leslie, L. L. (1997). Academic capitalism: Politics, policies, and the entrepreneurial university. Baltimore: The Johns Hopkins University Press.

Van der Vaart, W. (2017). Appreciative peer review: a procedure. AI Practitioner, 19(4), 6078.

Watkins, J.M., Mohr, B.J., \& Kelly, R. (2011). Appreciative Inquiry: change at the speed of imagination. Pfeiffer: San Fransisco. 
Yiend, J., Weller, S., \& Kinchin, I. (2014). Peer observation of teaching: The interaction between peer review and developmental models of practice. Journal of Further and Higher Education, 38(4), 465-484. 


\section{University Library}

\section{- M M I E E R VA A gateway to Melbourne's research publications}

Minerva Access is the Institutional Repository of The University of Melbourne

Author/s:

Fileborn, B;Wood, M;Loughnan, C

Title:

Peer reviews of teaching as appreciative inquiry: learning from "the best" of our colleagues

Date:

2020-11-03

Citation:

Fileborn, B., Wood, M. \& Loughnan, C. (2020). Peer reviews of teaching as appreciative inquiry: learning from "the best" of our colleagues. Higher Education, 83 (1), https:// doi.org/10.1007/s10734-020-00637-9.

Persistent Link:

http://hdl.handle.net/11343/249366 